\title{
GPPS-BJ-2019-0101
}

\section{Modelling Turbulent Transport Effects on the Formation Probability of Flame Kernel in Ignition Process}

\author{
Qing Xie, Zhuyin Ren \\ School of Aerospace Engineering, \\ Tsinghua University \\ jieq14@mails.tsinghua.edu.cn \\ Beijing, China
}

\author{
Hongjun Lin, Shoutang Shang \\ AECC Shenyang Engine Research Institute \\ nfsc2016@163.com \\ Shenyang, Liaoning, China
}

\author{
Ke Wang \\ Aero Engine Academy of China (AECC) \\ Joshua_wang@hotmail.com \\ Beijing, China
}

\author{
Wei Xiao \\ AECC Hunan Aviation Powerplant Research \\ Institute \\ Zhuzhou, Hunan, China
}

\begin{abstract}
In this study, the one-dimensional model for kernel formation accounting for turbulent transport (Kong et al., 2019) is first calibrated by the experimental data of turbulent expanding spherical flames of premixed ethylene/air mixtures. It further reveals that the ranges of equivalence ratio for successful kernel formation in turbulent flows can be much narrower than that in laminar flows, and in general, turbulent transport has more pronounced effects on fuel rich mixtures. In addition, the range decreases with increased turbulent intensity. The dependence of flammability limits for successful kernel formation on turbulent intensities is thereafter tabulated for methane/air flames and serves as the flammability criterion for kernel formation in a methane/air bluff-body flame. The kernel formation probability map for the bluff-body flame is computed with the flow and fuel/air mixing characteristics being extracted from a non-reacting Reynolds-Averaged Navier-Stokes (RANS) simulation. Results show that the computed probability map agrees qualitatively with experimental results, and it confirms that the turbulence has pronounced inhibitive effect on the formation of flame kernel before the stage of flame propagation in the ignition process.
\end{abstract}

\section{INTRODUCTION}

The development of advanced low-emission gas turbine combustor such as those in aero-engines demands more careful design of ignition devices and their placement in the combustion chamber to ensure reliable ignition or relighting in case of flame blow-out particularly at high altitudes. It is of practical interest to investigate physiochemical processes in the ignition process of gas turbine combustion and thereafter develop physical models and numerical approaches for the ignition prediction.

Four stages can be generally identified in a successful ignition event with electric spark (Mastorakos,2009; Eyssartier, 2013): (1) energy deposition, (2) formation of a viable flame kernel, (3) flame kernel growth and flame propagation, (4) flame stabilization. The forced ignition process in laboratory turbulent flames and gas turbine combustor have been extensively studied experimentally and numerically, showing that the forced ignition process is highly transient in nature and is intrinsically stochastic. Various factors can affect the development of spark kernels from the moment of energy deposition until the complete flame establishment. The most favourable ignition spots for sparking are influenced not only by local equivalence ratio, but also by the time history of the velocity fluctuations. The establishment of an ignition probability map for practical burner by experimental means is practically challenging and expensive.

Unsteady three-dimensional simulations could fairly describe the associated physics and give insights into the experimentally observed ignition phenomenon. Numerical work has explored various aspects of the behaviour of the kernel and the subsequent flame evolution with gaseous fuels (Chakraborty and Mastorakos, 2006, 2008) and liquid fuels (Neophytou, et al., 2010, 2011). For example, large eddy simulation (LES) have been performed to study the transient ignition process in laboratory flames such as methane/air bluff-body flames (Ahmed et al., 2007) and jet flames (Ahmed and Mastorakos, 2006). The ignition sequence has been studied in a full annular combustion chamber of gas turbine by means of massively parallel LES computations (Boileau et al., 2008). However, it is still impractical to compute the ignition 
probability map via repeating multiple 3D unsteady simulations to meet the design need of the ignition device in gas turbine combustors.

In practice, local ignition criteria and reduced models have been developed for different stages of ignition process (Neophytou et al., 2012; Eyssartier et al., 2013). In conjunction with the flow, turbulence and fuel/air mixing data that are extracted from (unsteady) nonreacting simulations, the ignition probability map could be computed. For example, five criteria that considers flammability, energy discharge, flame kernel growth, wall quenching, and upstream flame propagation respectively, have been employed to compute the ignition probability map of a turbulent two-phase flame (Eyssartier et al., 2013). The flame propagation criterion is to check if flame stabilization can be achieved by comparing the local flame speed and the flow field. In contrast, the criteria of flammability, and energy discharge etc., are employed to compute the probability for the kernel formation stage (Neophytou et al., 2012). For flame propagation and stabilization, the Lagrangian particle tracking method is employed, in which flame particles are tracked throughout the flow domain. The local Karlovitz number is computed and used as the criteria to check if the flame particles can ignite the regions they passed through. With this two-stage approach, Neophytou et al. studied the ignition process of a methane/air bluff-body flame, in which the flow and fuel/air mixing information is extracted from the LES simulation of nonreacting flow (Triantafyllidis et al., 2009). Results show that the reduction of ignition probability in the recirculation zone and the high ignition probability on the shear layer of the recirculation zone near the mean stoichiometric surface are well captured.

For both aforementioned approaches, the flammability criteria for kernel formation does not account for the effects of turbulent transport, which may not be appropriate at highly turbulent conditions since turbulent transport may inhibit the formation of a viable flame kernel before the stage of flame propagation. Recently, Ren et al, developed a reduced model for flame kernel formation (Kong et al., 2019), in which turbulent scalar transport during flame kernel formation is modelled through the incorporation of turbulence-induced diffusion in the different regions of the flame kernel. This reduced model can be employed to compute the flammability under different levels of turbulent intensity and therefore reflects the change of flammability of the gas mixture due to turbulent transport.

In this study, the reduced model is first calibrated by the experimental data of turbulent expanding spherical flames and is then used to determine the dependence of flammability limits of the methane/air mixtures for successful kernel formation on different levels of turbulent intensities. Then these flammability limits are employed to investigate the effect of turbulent transport for kernel formation probability in a methane/air bluff-body flame, with the flow and fuel/air mixing characteristics being extracted from a non-reacting RANS simulation.
THE ONE-DIMENSIONAL MODEL FOR KERNEL FORMATION ACCOUTING FOR THE TURBULENT TRANSPORT

\section{Model description}

In the 1D model (Kong et al., 2019), the governing equations in spherical coordinates are

$$
\begin{gathered}
\frac{\partial \rho}{\partial t}+\frac{1}{r^{2}} \frac{\partial}{\partial r}\left(r^{2} \rho u_{r}\right)=0 \\
\rho \frac{\partial Y_{k}}{\partial t}+\rho u_{r} \frac{\partial Y_{k}}{\partial r}=\frac{1}{r^{2}} \frac{\partial}{\partial r}\left(r^{2} \rho D_{k}^{*} \frac{\partial Y_{k}}{\partial r}\right)+\dot{\omega}_{k}(p, T, \boldsymbol{Y}) \\
\rho c_{p} \frac{\partial T}{\partial t}+\rho c_{p} u_{r} \frac{\partial T}{\partial r}=\frac{1}{r^{2}} \frac{\partial}{\partial r}\left(r^{2} \rho \lambda^{*} \frac{\partial T}{\partial r}\right)-\sum_{k=1, N} \dot{\omega}_{k} h_{k},
\end{gathered}
$$

where $\rho, p, u_{r}, Y_{k}, T$ denote the density, pressure, velocity, mass fraction of species $k$ and temperature, $c_{p}$ is the massaveraged specific heat, $D_{k}^{*}$ and $\lambda^{*}$ are the effective diffusivity and conductivity, and $\dot{\omega}_{k}$ and $h_{k}$ denotes the net creation rates and enthalpy of $k t h$ species. The reaction source term $\dot{\omega}_{k}$ is evaluated directly using the computed mean composition in conjunction with detailed chemical kinetics.

The effective diffusivity $D_{k}^{*}$ and conductivity $\lambda^{*}$ are modelled as

$$
\begin{aligned}
D_{k}^{*} & =D_{k}+D_{\text {turb }}(r, t), \\
\lambda^{*} & =\lambda+\lambda_{\text {turb }}(r, t),
\end{aligned}
$$

where $D_{k}$ and $\lambda$ are the laminar diffusivity and conductivity, respectively; $D_{\text {turb }}$ and $\lambda_{\text {turb }}$ are the turbulent diffusivity and conductivity varying with time and position, respectively. In this study, the laminar transport properties are computed based on the local temperature and composition with mixture-averaged formula using CHEMKIN (Kee et al., $1989)$. With a unity turbulent Lewis number, $\lambda_{\text {turb }}$ is related to $D_{\text {turb }}$ through

$$
\lambda_{\text {turb }}=\rho c_{p} D_{t u r b} .
$$

The turbulent diffusivity, $D_{\text {turb }}$, which represents smallscale turbulence induced diffusion during the flame kernel formation, is modelled using the idea of residual eddy viscosity (Pope, 2000)

$$
D_{\text {turb }}=\frac{C_{0}}{\pi^{2 / 3}} \Delta^{4 / 3} u^{\prime} / l_{t}^{1 / 3},
$$

where $C_{0}$ is a model constant, $u^{\prime}$ and $l_{t}$ are turbulent fluctuation velocity and turbulence integral length scale respectively. Note that $\Delta$ is a zone-dependent characteristic length scale with the 1D spherical flame structure being divided into the post flame zone, reaction zone, preheat zone and unburnt zone, respectively. For turbulent flames in thin reaction zones regime, turbulence has no direct impact on reaction zone and the associated turbulent diffusivity is zero in this zone. For the preheat and post-flame zones, the transport effect of turbulence eddies with sizes being comparable or smaller than the corresponding zone thickness is modelled using Eq. (7) with individual characteristic lengthscale. For the unburnt zone, the modelling for turbulent diffusivity is inessential since there is no scalar gradient in this zone. 
In this study, the flame structure is parameterized based on the progress variable $c$, which is defined based on the fraction of carbon in species $\mathrm{CO}$ and $\mathrm{CO}_{2}$ over that in all the carbon-containing species. The boundaries between different zones and the model constant $C_{0}$ are determined through the calibration of predicted flame propagation with experimental data.

\section{Calibration of the reduced model}

The model parameters are first calibrated using the experiment results of outwardly propagating turbulent ethylene/air flames under different turbulent intensities, which are performed in the constant-pressure dual-chamber spherical flame apparatus at Tsinghua University (Huo et al., 2018). Details of the apparatus and the experiments have been described (Huo et al., 2018). The turbulence in the chamber is generated through eight uniformly distributed electric fans. The averaged magnitude of the turbulent fluctuation velocities, $u^{\prime}$, and corresponding fan speeds, $n$, employed in this work for model calibration, are listed in Table 1. The turbulence integral length scale in the experiments is about $1 \mathrm{~cm}$.

Table 1. The turbulent fluctuation velocities and corresponding fan speeds employed for calibration.

\begin{tabular}{ccc}
\hline Case ID & $u^{\prime}(\mathrm{m} / \mathrm{s})$ & $n(\mathrm{rpm})$ \\
\hline 1 & 2.3 & 3000 \\
\hline 2 & 4.0 & 5000 \\
\hline
\end{tabular}

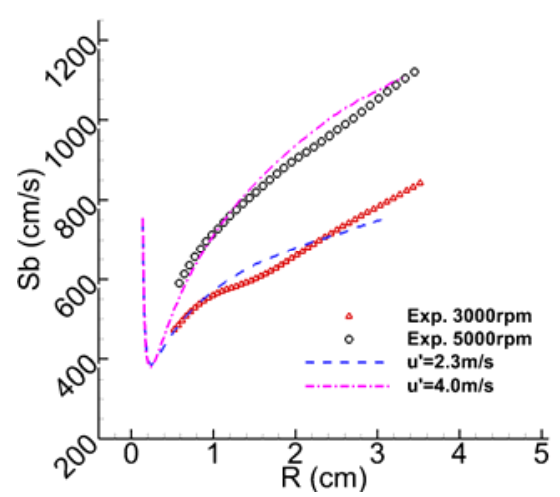

Figure 1 The evolution of flame speed ( $\mathrm{Sb}$ ) vs. flame size (R) in experiments (dots) and simulations (dash and dash dot lines).

The ethylene mechanism (Luo et al., 2012) with 32 species and 206 reactions is used in the calculation. The model constant, $C_{0}$, is set to be unity. The input turbulence integral length scale, $l_{t}$ is set to be $1 \mathrm{~cm}$. The calibrated boundaries among post flame zone, reaction zone, preheat zone and unburnt zone are at $c_{1}=0.985, c_{2}=0.5$, and $c_{3}=0.02$, respectively. Figure 1 shows the evolution of flame speed with flame radius under two representative turbulent conditions that are featured by the fan speeds of 3000rpm and 5000rpm in experiments and by the input turbulent fluctuation speeds of $2.3 \mathrm{~m} / \mathrm{s}$ and $4.0 \mathrm{~m} / \mathrm{s}$ in simulations. The flame speeds in experiments are reconstructed based on the evolution of instantaneous flame area that is imaged using Schlieren photography and recorded with a high-speed digital camera (Huo et al., 2018). As shown, the predicted flame propagation processes agree well with those from the experimental ones. Particularly, the early stage, i.e., when the flame radius is smaller than $1 \mathrm{~cm}$, is well captured, demonstrating that the $1 \mathrm{D}$ model can accurately capture the kernel formation process with various levels of turbulent intensity.

\section{KERNEL FORMATION PROBABILITY MAP FOR A METHANE/AIR BLUFF-BODY FLAME}

\section{Criteria for the kernel formation}

The following three criteria for kernel formation, suitable for two-phase flows with liquid fuel, are employed (Eyssartier et al., 2013). The first one is that the equivalence ratio must be within the flammability limits,

$$
\phi_{\text {low }} \leq \phi \leq \phi_{\text {up }}
$$

with the reduced model, $\phi_{\text {low }}$ and $\phi_{u p}$ can be flow dependent and therefore account for the effect of turbulent transport on flammability.

The second criterion is that the energy deposit must at least increase the unburnt temperature $T_{u n}$ of gas mixture to its ignition temperature $T_{i g n}$ and bring the temperature of cold liquid fuel to its saturation temperature

$$
\frac{E_{d e p}}{\rho C_{p}} \geq\left(T_{i g n}-T_{u n}\right)+\left(\alpha_{l}+\frac{4 n_{d i m}}{3 N u} \frac{d_{l}^{2}}{d_{k e r}^{2}}\right) \frac{\rho_{l} C_{p, l}}{\rho C_{p}}\left(T_{c c}-T_{l, \infty}\right)
$$

where, the ignition temperature $T_{i g n}$ of the gas mixture depends on equivalence ratio. In this study it is tabulated from the results of homogeneous auto-ignition simulations. The ignition temperature is defined so that the ignition delay time under such initial temperature in auto-ignition simulation should be smaller than $1 \mathrm{~ms}$, which is some representative timescale in gas turbine combustors.

The third criterion requires that the thermal diffusion to the surrounding gas must not be faster than liquid fuel vaporization, i.e.,

$$
t_{\text {vap }} \leq t_{\text {diff }}
$$

With the three criteria for flame kernel formation, the probability map of kernel formation in turbulent flames can be computed through the following three steps. First, with the mean mixture fraction and its variance being extracted from a non-reacting simulation, a beta probability density distribution function is used to generate many realizations of fuel/air mixtures for each location, which is 100 in this work. Then, the criteria are applied for individual samples at each location of the cold flow. Finally, the percentage of events satisfying the criteria for successful kernel formation is counted to form the probability map of kernel formation.

Note that for the gaseous methane/air bluff-body flame considered in this study, only the first two criteria are effective. It is further noted that the energy deposit for ignition is sufficiently high and the second criterion is always guaranteed.

\section{Flammability limits of methane/air mixtures}


For the methane/air bluff-body flame as sketched in Fig. 2 , the calibrated 1D model is used to predict the flammability limits for methane/air mixture under different turbulent intensities. The methane mechanism with 25 species and 125 reactions is used (Zhou et al., 2017). Figure 3 shows the ignition events for methane/air flames under different values of $u^{\prime}$ and $\phi$. The lower and upper limits for laminar case, in equivalence ratios, are about $\phi_{\text {low }}=0.5$ and $\phi_{u p}=1.6$, or in mixture fractions $\xi_{\text {low }}=0.0283$ and $\xi_{\text {up }}=0.0853$, which are close to the reported values 0.028 and 0.089 in (Ahmed et al., 2007). Note that, currently, the ignition in a simulation is deemed "failure" if the flame kernel radius decreases after a sufficiently long time. In the simulation, a hot spot of $2000 \mathrm{~K}$ with $1 \mathrm{~mm}$ in radius, whose energy is higher than the minimum ignition energy, is used to initiate the flows.

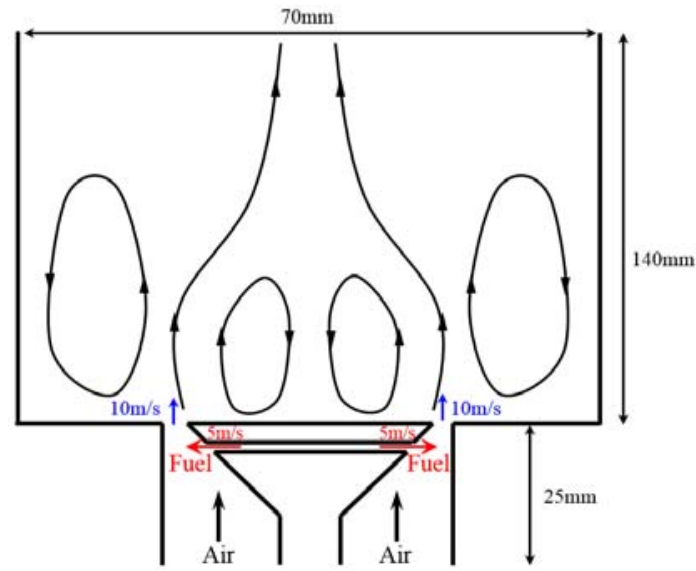

Figure 2. Schematic of the bluff-body flame, showing the inlet of fuel and air, and the recirculation zones.

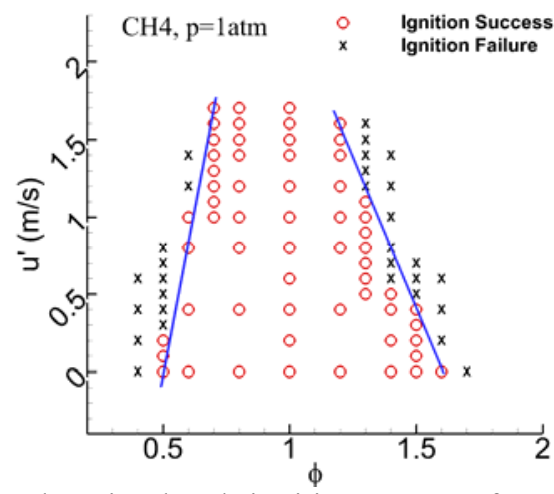

Figure 3. The simulated ignition events for methane/air spherical flames at $p=1$.0atm under various $u^{\prime}$ and $\phi$.

The effects of turbulent transport on the flammability limits, can be parameterized by the linear dependence of the limits, $\phi_{\text {low }}$ and $\phi_{u p}$, on turbulent fluctuation velocity, $u^{\prime}$. In this study, the change of flammability limits is characterised by the four controlling limits at $u^{\prime}=0 \mathrm{~m} / \mathrm{s}$ and $u^{\prime}=1.6 \mathrm{~m} / \mathrm{s}$, respectively. Different slopes of changes are considered for parametric studies of the turbulent transport effects on kernel formation probability. Specifically, the four cases of the control points are listed in Table 2.
Table 2. Selected sets of control points $\left(u^{\prime}, \phi\right)$ for the functions of flammability limits

\begin{tabular}{ccc}
\hline Case ID & $\phi_{\text {low }}$ & $\phi_{\text {up }}$ \\
\hline \multirow{2}{*}{1} & $(0.0,0.5)$ & $(0.0,1.6)$ \\
& $(1.6,0.7)$ & $(1.6,1.2)$ \\
\hline \multirow{2}{*}{2} & $(0.0,0.5)$ & $(0.0,1.6)$ \\
& $(1.6,0.7)$ & $(1.6,1.1)$ \\
\hline \multirow{2}{*}{3} & $(0.0,0.5)$ & $(0.0,1.6)$ \\
& $(1.6,0.7)$ & $(1.6,1.3)$ \\
\hline \multirow{2}{*}{4} & $(0.0,0.5)$ & $(0.0,1.6)$ \\
& $(1.6,0.6)$ & $(1.6,1.2)$ \\
\hline
\end{tabular}

Kernel formation probability map in the methanelair bluffbody flame

The steady state RANS simulation of the methane/air bluff-body flame, the case " $\mathrm{B}$ " in (Ahmed et al., 2007), is performed using the commercial software ANSYS Fluent. The schematic of the flame is shown in Figure 2. The standard $\mathrm{k}-\varepsilon$ turbulence model and the partially premixed combustion model (with chemical reactions being turned off) are used to predict the turbulent flow and mixing fields. Specifically, the transport equations for mixture fraction and its variance are solved to compute the fuel/air mixing fields. The mean and variance of mixture fraction are needed for the generation of realizations in conjunction with the beta PDF for computing kernel formation probability.

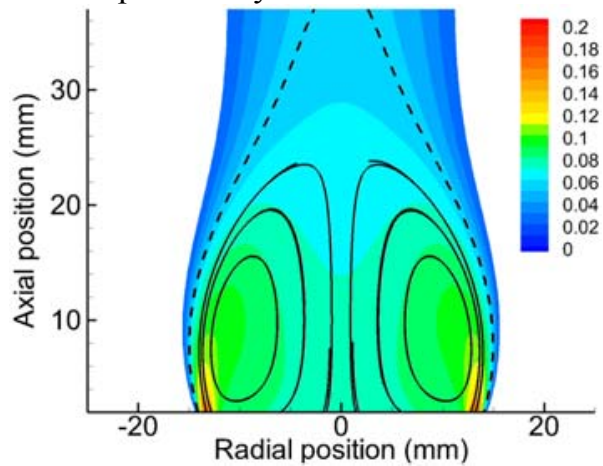

(a)

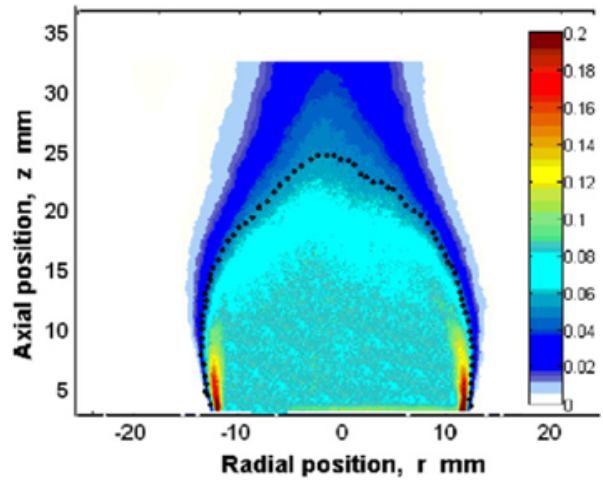

(b)

Figure 4. Mean mixture fraction. (a) from the RANS. (b) from the experiment, reproduced from Fig. 9(b) in (Ahmed et al., 2007). The dashed line marks the iso-surface of stoichiometric mean mixture fraction. The recirculation zone is marked by representative streamlines. 
The computational domain is sketched in Figure 2. The upstream of the pipe is set to be the velocity inlet of air, and the inlet velocity is adjusted to ensure that the velocity at the exit of the bluff-body is $10 \mathrm{~m} / \mathrm{s}$. The fuel nozzle is set to be a velocity inlet with the velocity of $5 \mathrm{~m} / \mathrm{s}$. All the other model surfaces are set to be non-slipping walls. The downstream exit is set to be outlet boundary. The domain is meshed with structured grid with 6.28 million cells. The grid is refined in the recirculation zone with a resolution of $0.5 \mathrm{~mm}$.

Figure 4 shows the mean mixture fraction from numerical and experiment results, respectively. As shown, the computed iso-surface of stoichiometric mixture fraction is much elongated along the axis line. In addition, the simulation yields much higher mixture fractions in the recirculation zone. This has pronounced effect on the prediction of kernel formation probability as shown below. Further improvement for the nonreacting simulation is subject to future study.

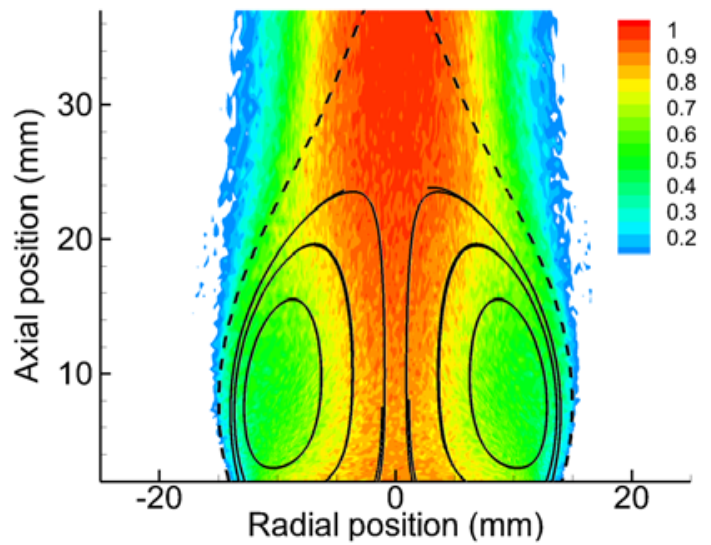

(a) Kernel formation probability

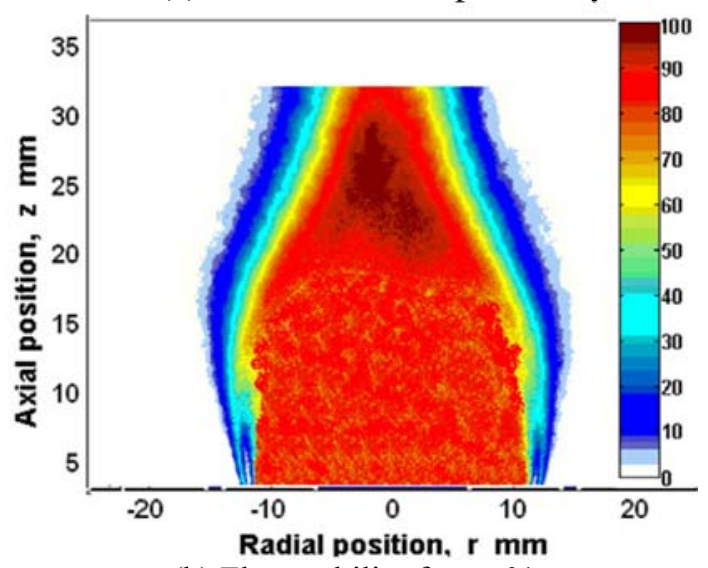

(b) Flammability factor $\%$

Figure 5. (a) Kernel formation probability predicted by the flammability criterion with laminar flammability limits. (b) Flammability factor from experiment, reproduced from Fig. 22(c) in (Ahmed et al., 2007). The dashed line marks the isosurface of mean stoichiometric mixture fraction.

Figure 5 shows the kernel formation probability without considering the effects of turbulent transport on the flammability limits. Compared with the flammability factor from the experiment, the calculated probability is much lower than that from experiment in the recirculation zone. This is due to the fact that the predicted mean mixture fraction $(>0.09)$ in recirculation zone is much higher than that from the experiment and is larger than the upper flammability limit 0.089. Note that, when computing the kernel formation probability, the second and third criteria introduced previously take no effect due to the use of high spark energy and gaseous fuel in this work.

The results from the parametric study of turbulent transport effect on kernel formation probability are shown in Fig. 6. Note that Fig. 6(a) shows the ignition probability map for the whole ignition process. For the base case, case-1, it can be seen that the kernel formation probabilities in the recirculation zone agrees with the ones from experiment. This confirms the argument that turbulent fluctuations in the recirculation zone indeed inhibit the formation of a viable flame kernel before the stage of flame propagation and can reduce the ignition probability. The large difference in the probabilities for the downstream of the recirculation zone between Fig. 6(a) and (b) is not surprising since the current work mainly focuses on the formation of flame kernel and has not account for the flame propagation and stabilization stage. For the downstream region outside the recirculation zone, flame cannot be stabilized even a viable flame kernel can be formed.

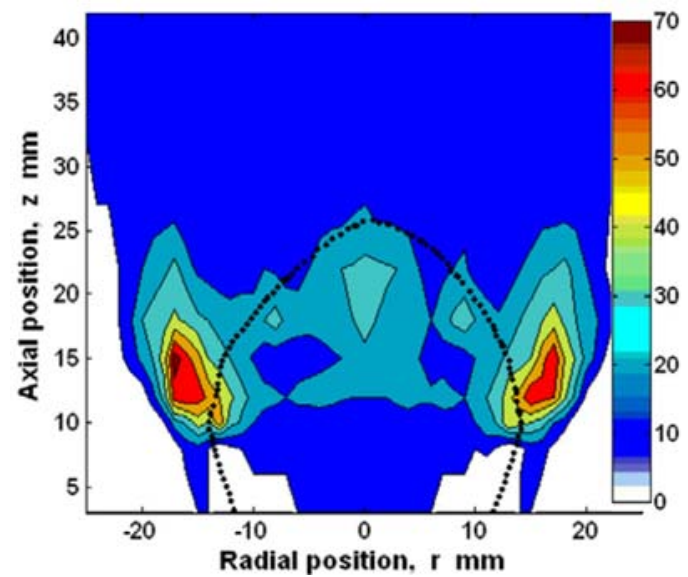

(a) Ignition probability from experiment (Ahmed et al., 2007).

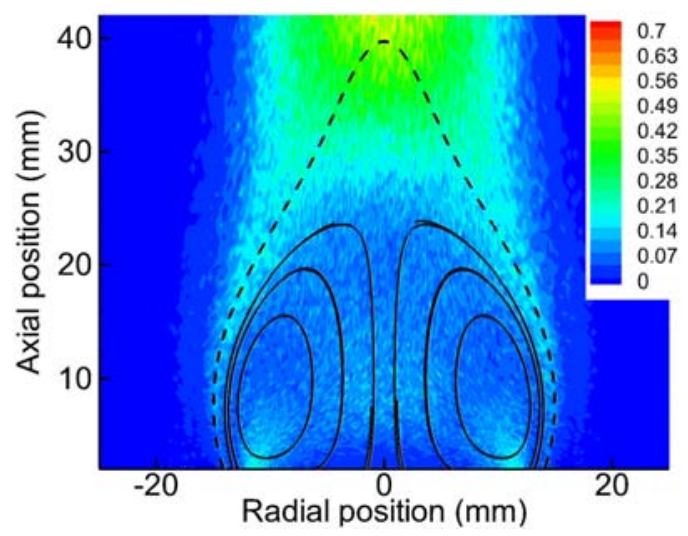

(b) Kernel formation probability, Case-1. 


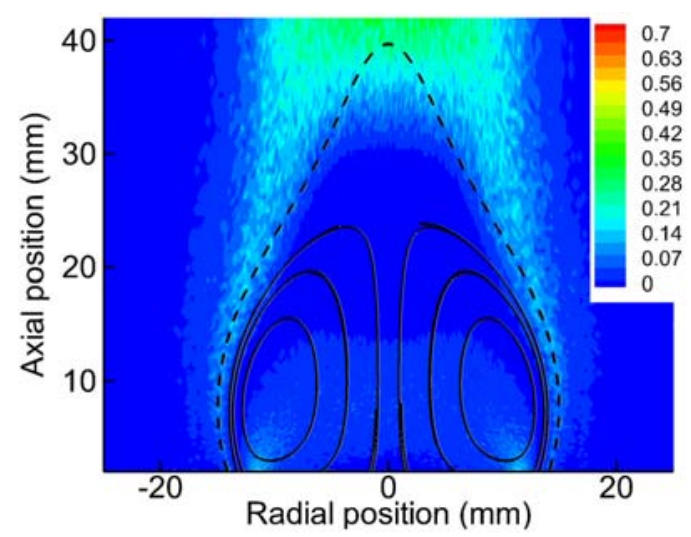

(c) Kernel formation probability, Case-2.

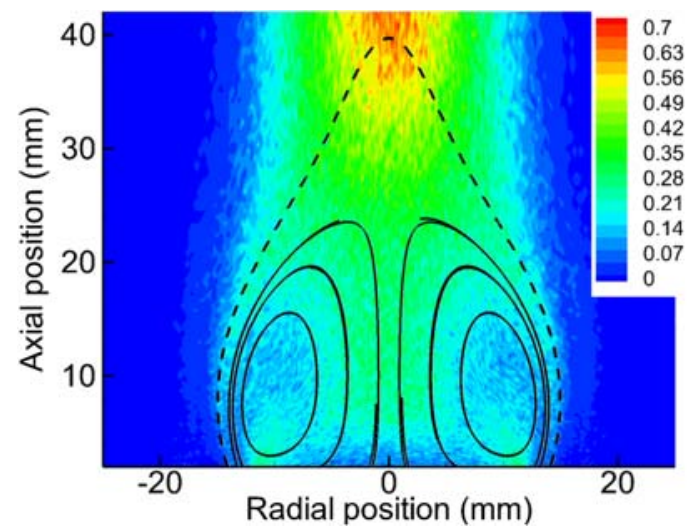

(d) Kernel formation probability, Case-3.

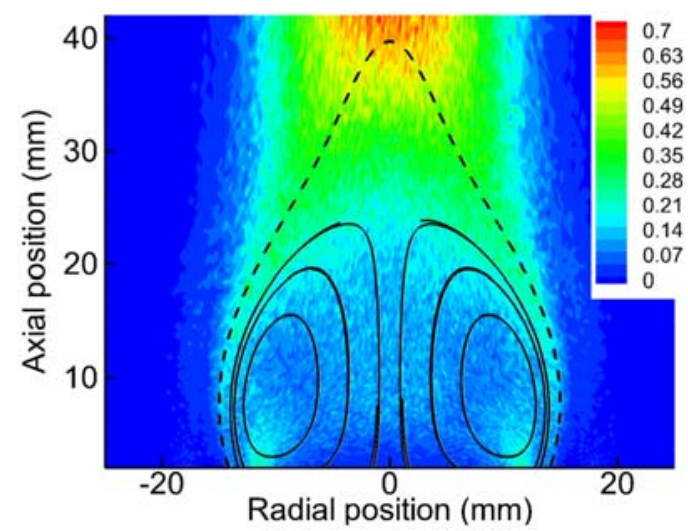

(e) Kernel formation probability, Case-4.

Figure 6. (a) The ignition probability from experiment, reproduced from Fig. 22(a) in (Ahmed et al., 2007), (b-e) kernel formation probability predicted by the flammability criterion with different parameterization of turbulent effects.

The parametric study of the upper flammability limits (see case 1, 2 and 3 in Figures $6 \mathrm{~b}, 6 \mathrm{c}$ and $6 \mathrm{~d}$ ), the kernel formation probability in the recirculation zone is more sensitive to the change of the upper flammability limit by turbulence. This is due to the fact that the predicted mixture fraction in the recirculation zone is relatively high. The large change of the upper flammability limit by turbulence can thereafter alter the kernel formation probability. In contrast, the kernel formation probability is not so sensitive to the change of lower limit of the flammability by turbulence. (see Figure 6e). It is worth mentioning that the likelihood of high kernel formation probability near the shear layer of the recirculation zone has also been captured and this agrees with experiment qualitatively. In addition, the likelihood of high kernel formation probability in the shear layer is insensitive to the parameterization of turbulent transport effects.

\section{CONCLUSIONS}

In this study, a method has been formulated to compute the kernel formation probability in an ignition process, in which the effect of turbulent transport on the flammability limits for kernel formation is accounted for through the incorporation of turbulence-induced diffusion in the different regions of the 1-D spherical flame kernel model. The model is first calibrated with turbulent premixed ethylene/air expanding spherical flames to yield the correct flame propagation speeds under various levels of turbulent intensities. The calibrated model has then been employed to compute the flammability limits of methane/air mixture at different turbulent intensity. It is found that the computed flammability limits at laminar conditions match the experimental ones. It further reveals that turbulent transport can significantly narrow the flammability ranges for successful kernel formation and it has more pronounced effect on the fuel rich flammability limit.

These flammability limits accounted for turbulent transport are used to compute the kernel formation probability of a methane/air bluff-body flame with the flow and fuel/air mixing characteristics being extracted from a nonreacting steady state RANS simulation. Compared to the experimental data, the nonreacting simulation yields elongated stoichiometric iso-surface in the flow direction and also slightly larger mixture fraction in the recirculation zone, which is subject to further improvement. The parametric study shows that the turbulent transport can inhibit the formation of flame kernel before the stage of flame propagation. The computed kernel formation probability agrees with experimental result in the recirculation zone. The likelihood of high kernel formation probability near the shear layer of the recirculation zone has also been captured and this agrees with experiment qualitatively. Further improvement in the kernel formation probability relies on more accurate prediction of the flow and mixing fields in the nonreacting simulations.

\section{ACKNOWLEDGMENTS}

This work is supported by National Natural Science Foundation of China No.91841302 and No.91641205.

\section{REFERENCES}

[1]. Ahmed S. F., Balachandran R., Marchione T., Mastorakos E. (2007). Spark ignition of turbulent nonpremixed bluff-body flames. Combustion and Flame, 151(1-2), 366-385.

[2]. Ahmed S. F., Mastorakos E. (2006). Spark ignition of lifted turbulent jet flames. Combustion and Flame, 146(1-2), 215-231.

[3]. Boileau M., Staffelbach G., Cuenot B., Poinsot T., Bérat C. (2008). LES of an ignition sequence in a gas turbine engine. Combustion and Flame, 154(1-2), 2-22. 
[4]. Chakraborty N., Mastorakos E. (2006). Numerical investigation of edge flame propagation characteristics in turbulent mixing layers. Physics of Fluids, 18(10), 105 103.

[5]. Chakraborty N., Mastorakos E. (2008). Direct numerical simulations of localised forced ignition in turbulent mixing layers: the effects of mixture fraction and its gradient. Flow Turbulence and Combustion, 80(2), 155186.

[6]. Eyssartier A., Cuenot B., Gicquel L. Y. M., Poinsot T. (2013). Using LES to predict ignition sequences and ignition probability of turbulent two-phase flames. Combustion and Flame, 160(7), 1191-1207.

[7]. Huo J., Yang S., Ren Z., Zhu D., Law C.K. (2018). Uncertainty reduction in laminar flame speed extrapolation for expanding spherical flames. Combustion and Flame, 189, 155-162.

[8]. Kong L., Ren, Z., Hou, L. (2019). Modeling turbulent transport effects on kernel formation and flame propagation in an ignition process. Chinese Journal of Aeronautics, 32(4), 895-905.

[9]. Kee R. J., Rupley F. M., Miller J. A. (1989). ChemkinII: A Fortran chemical kinetics package for the analysis of gas-phase chemical kinetics. Sandia National Labs., Livermore, CA (USA).

[10]. Lacaze G., Richardson E., Poinsot T. (2009). Large eddy simulation of spark ignition in a turbulent methane jet. Combustion and Flame, 156(10), 1993-2009.

[11]. Luo Z., Yoo C. S., Richardson E. S., Chen J. H., Law C. K., Lu T. (2012). Chemical explosive mode analysis for a turbulent lifted ethylene jet flame in highly-heated coflow. Combustion and Flame, 159(1), 265-274.

[12]. Mastorakos E. (2009). Ignition of turbulent nonpremixed flames. Progress in Energy and Combustion Science, 35(1), 57-97.

[13]. Neophytou A., Mastorakos E., Cant R. S. (2010). DNS of spark ignition and edge flame propagation in turbulent droplet-laden mixing layers. Combustion and Flame, 157(6), 1071-1086.

[14]. Neophytou A., Mastorakos E., Cant R. S. (2011). Complex chemistry simulations of spark ignition in turbulent sprays. Proceedings of the Combustion Institute, 33(2), 2135-2142.

[15]. Neophytou A., Richardson E. S., Mastorakos E. (2012). Spark ignition of turbulent recirculating non-premixed gas and spray flames: A model for predicting ignition probability. Combustion and Flame, 159(4), 1503-1522.

[16]. Pope S. B. (2000). Turbulent flows. Cambridge University Press.

[17]. Sitte M. P., Bach E., Kariuki J., Bauer H., Mastorakos E. (2016). Simulations and experiments on the ignition probability in turbulent premixed bluff-body flames. Combustion Theory and Modelling, 20(3), 548-565.
[18]. Subramanian V., Domingo P., Vervisch L. (2010). Large eddy simulation of forced ignition of an annular bluffbody burner. Combustion and Flame, 157(3), 579-601.

[19]. Triantafyllidis A., Mastorakos E., Eggels R. (2009). Large Eddy Simulations of forced ignition of a nonpremixed bluff-body methane flame with Conditional Moment Closure. Combustion and Flame, 156(12), 2328-2345.

[20]. Weckering J., Sadiki A., Janicka J., Mastorakos E., Eggels R. (2011). A forced ignition probability analysis method using LES and Lagrangian particle monitoring. Proceedings of the Combustion Institute, 33(2), 29192925.

[21]. Zhou H., Li S., Ren Z., Rowinski D. H. (2017). Investigation of mixing model performance in transported PDF calculations of turbulent lean premixed jet flames through Lagrangian statistics and sensitivity analysis. Combustion and Flame, 181, 136-148. 